\title{
Clinical outcomes of fast-track total knee arthroplasty for patients aged $>80$ years
}

\author{
TP Leung *, CH Lee, Esther WY Chang, QJ Lee, YC Wong
}

\section{A B S T R A C T}

Introduction: Because of the ageing population in Hong Kong, there is an increasing incidence of total knee arthroplasties (TKA) conducted in patients aged $>80$ years.

Methods: This retrospective case-control study enrolled all patients who were aged $>80$ years and underwent fast-track primary TKA between 2011 and 2015. Their outcomes were compared with the outcomes of a matched control group of younger patients who underwent fast-track TKA in the same period.

Results: In total, 220 patients were included in this study with a follow-up period of at least 2 years (mean=3.2 years; range, 2-5 years); 112 (51\%) were octogenarians and 108 (49\%) were nonoctogenarians. Greater improvement in Knee Society Score was found in the octogenarian group at 1 year after surgery $(46 \pm 19$ vs $39 \pm 16, P=0.018)$. The incidence of complications was higher in the octogenarian group $(15.2 \%$ vs $4.6 \%, \mathrm{P}=0.009)$. There were no significant differences in the incidence of

This article was published on $18 \mathrm{Feb}$ 2022 at www.hkmj.org. major complications, the rate of intensive care unit admission, or the 1-year mortality rate between

the two groups. After adjustment for confounding factors, Charlson Comorbidity Index $>5$, history of major cerebrovascular accident, and history of peptic ulcer disease were predictive of complications after fast-track TKA $(\mathrm{P}=0.039, \mathrm{P}=0.016$, and $\mathrm{P}=0.007$, respectively); octogenarian status was not predictive of complications.

Conclusions: Octogenarians had greater improvement in Knee Society Score at 1 year after fast-track TKA, compared with non-octogenarians, but there were no significant differences in the incidences of mortality or major complications.

\section{Hong Kong Med J 2022;28:7-15 \\ https://doi.org/10.12809/hkmj208911}

TP Leung *, MB, ChB

CH Lee, FHKCOS, FHKAM (Orthopaedic Surgery)

EWY Chang, MSc

QJ Lee, FHKCOS, FHKAM (Orthopaedic Surgery)

YC Wong, FHKCOS, FHKAM (Orthopaedic Surgery)

Joint Replacement Centre, Yan Chai Hospital, Hong Kong

* Corresponding author: fos.markleung@gmail.com

New knowledge added by this study

- The octogenarian group exhibited greater improvement in functional outcomes after fast-track total knee arthroplasty, compared with younger patients.

- Charlson Comorbidity Index $>5$, history of major cerebrovascular accident, and history of peptic ulcer disease were predictive of complications after fast-track total knee arthroplasty.

Implications for clinical practice or policy

- Age alone should not be a contra-indication to total knee arthroplasty because there were no significant differences in major complications or mortality rate for patients aged $>80$ years who underwent fast-track total knee arthroplasty.

- Fast-track total knee arthroplasty could be useful for managing the growing osteoarthritis burden among older adults in Hong Kong, thus improving their quality of life.

\section{Introduction}

Because of the ageing population in Hong Kong, the incidence of total knee arthroplasty (TKA) for patients aged $>80$ years is expected to increase. ${ }^{1,2}$ The optimal age for arthroplasty has generally been regarded as between 60 and 80 years. Age was previously identified as an independent risk factor for mortality and major complications after TKA. For example, Kreder et $\mathrm{al}^{3}$ reported a 2.5 -fold increase in the risk of acute myocardial infarction and a 3.4-fold increase in mortality among octogenarians undergoing TKA, compared with a cohort aged 65 to 79 years. Arthroplasties in patients aged $>80$ years were declined by surgeons or patients because of the expected high rates of postoperative morbidity and mortality. ${ }^{4-6}$

With preoperative preparations intended to minimise perioperative complications and blood loss, a recent study suggested that TKA could be a safe procedure among patients aged $>80$ years. $^{7}$ The incidences of TKA in patients aged $>80$ years have been increasing in various knee registries. ${ }^{8-12}$ 


\section{0 歲以上患者快速全膝關節置換術的臨床結果}

\section{梁棣邦、李祥康、曾慧儀、李君哲、黃耀忠}

引言：由於香港人口老化，80歲以上患者接受全膝關節置換術 （TKA）的發生率越來越高。

方法：這項回顧性病例對照研究納入 2011 年至 2015 年間所有 80 歲以 上接受快速原發性TKA的患者, 並將他們的結果與同期接受快速TKA 的年輕患者對照組的結果進行比較。

結果：共納入 220 名患者, 隨訪時間至少 2 年（平均 3.2 年, 介平 $2-5$ 年)。當中, 耄荎組別佔 112 人 $(51 \%)$, 非耄荎組別佔 108 人 (49\%)。術後1年, 老薪組的膝關節協會評分有較大改善 $(46 \pm 19$ 比 $39 \pm 16, \mathrm{P}=0.018)$ 。老葟組的併發症發生率較高（15.2\%比 $4.6 \%, \mathrm{P}=0.009)$ 。兩組的嚴重併發症發生率、重症監護病房入院率 或 1 年死亡率無顯著差異。調整混雜因素後, Charlson併發症指數 5 以 上、重大腦血管意外病史和消化性潰瘍病史可預測快速TKA的術後併 發症（分別為 $\mathrm{P}=0.039 、 \mathrm{P}=0.016$ 和 $\mathrm{P}=0.007$ ）。老荎狀況不能預測併 發症。

結論：與非耄薪組相比, 老荎組在快速TKA術後 1 年的膝關節協會評 分有更大改善, 但死亡率或嚴重併發症的發生率沒有顯著差異。

However, the traditional approach of declining knee arthroplasties in patients aged $>80$ years remains popular in Hong Kong. This approach does not meet the increasing needs of the ageing population.

The current study investigated whether octogenarians could achieve similar clinical outcomes after TKA, compared with younger patients. The null hypothesis was that short-term function and complications would not significantly differ between patients aged $>80$ years and patients aged $\leq 80$ years after fast-track primary TKA.

\section{Methods}

\section{Study design and setting}

This retrospective case-control study was carried out in the Total Joint Replacement Centre in Yan Chai Hospital in Hong Kong between 2011 and 2015. The results of TKA procedures performed in the institute during the study period were reviewed; all patients were followed up for at least 2 years (mean $=3.2$ years; range, $2-5$ years).

\section{Study population}

All patients who were aged $>80$ years and underwent primary TKA during the study period were included in the analysis. Their outcomes were compared with the outcomes in a similar number of younger patients (aged $\leq 80$ years) with a matched sex ratio and body mass index (BMI). The indications for TKA were primary osteoarthritis of the knee and rheumatoid arthritis of the knee. The exclusion criteria in this study were revision TKA and simultaneous bilateral
TKA. The contra-indications for TKA in both groups included active local or remote infection, poor skin condition, recent stroke and myocardial infarction (ie, within 1 year), poor cardiopulmonary reserve (eg, congestive heart failure and chronic obstructive pulmonary disease), and cirrhosis. For patients who had undergone percutaneous coronary intervention involving dual antiplatelet therapy, TKA was delayed for 1 year.

\section{Data retrieval and measurement}

Data retrieval was performed using the Clinical Management System in our institute. The procedure code for retrieval was '81.54 total knee replacement'. All operative records and out-patient records were reviewed. All outcome measurements were performed by independent observers (ie, orthopaedic specialist nurses) who were blinded to the details of treatment.

\section{Baseline characteristic and outcome variables}

Baseline characteristics were compared between the two groups; these included age, sex, BMI, co-morbidities, Charlson Comorbidity Index, preoperative haemoglobin level, and type of anaesthesia. Primary outcome measures included the knee range of motion (ROM), Knee Society Score (KSS), Western Ontario and McMaster Universities Osteoarthritis Index (WOMAC), complication rates, and mortality rates. Secondary outcome measures included the need for blood transfusion, postoperative admission to the intensive care unit (ICU), length of hospitalisation , and postoperative ambulatory status.

\section{Fast-track protocol}

Using the fast-track protocol, a multidisciplinary approach was adopted in the perioperative period (Fig 1). Patients were examined in the pre-admission clinic by an orthopaedic surgeon, an anaesthetist, and an orthopaedic specialty nurse. Preoperative workups with blood tests (eg, complete blood count, liver function test, renal function test, random glucose, and haemoglobin), chest X-rays, and electrocardiography were performed in the pre-admission clinic. Blood pressure and BMI measurements were also conducted in the preadmission clinic; blood pressure $<160 / 90 \mathrm{~mm} \mathrm{Hg}$ was required. The presence of local skin problems and other acute infections (eg, skin, oral, or urinary tract) were ruled out. Previous histories of deep vein thrombosis (DVT), bleeding tendency, gastrointestinal bleeding, and haemorrhagic stroke were recorded. Finally, other pre-existing medical conditions (eg, thyroid disease and obstructive sleep apnoea) were evaluated in the pre-admission clinic. The anaesthetist carried out a preoperative 
assessment and determined the anaesthesia modality (general or spinal); spinal anaesthesia was preferred unless contra-indicated. If patients had poorly controlled hypertension and diabetes, they were referred to a fast-track pathway for assessment by a family physician to optimise and titrate medical therapy for hypertension and diabetes. Fast-track echocardiography was also conducted with support from cardiologists to assess baseline cardiac function and suspected valvular problems. After patients had received explanations of TKA, as well as its benefits and risks, they provided written informed consent to undergo the surgical procedure.

Education to manage patient expectations was conducted by a nursing specialist. Prior to surgery, a physiotherapist provided patients with education concerning the rehabilitation pathway (ie, exercise, home care, and the arrangement of walking aids). An occupational therapist performed an Activities of Daily Living assessment and conducted appropriate home modifications. A medical social worker performed a psychosocial assessment and discharge planning; for patients with anticipated discharge problems, short-term placement was arranged prior to surgery. The aim of this multidisciplinary preoperative preparation protocol was to reduce the in-patient period and promote early postoperative ambulation for successful rehabilitation.

Most patients were admitted for same-day surgery. If the anaesthetist requested a short period of monitored preoperative optimisation, patients were admitted for next-day surgery. The criteria for next-day surgery included the presence of insulindependent diabetes mellitus requiring overnight dextrose-potassium-insulin infusion, the presence of chronic obstructive pulmonary disease, and the need for pacemaker adjustment prior to surgery.

\section{Surgical techniques and perioperative management}

All arthroplasties were performed via the medial parapatellar approach using a tourniquet, a posterior-stabilised implant, and a bone plug in the intramedullary canal or navigation without canal violation, followed by cementation, haemostasis with a tourniquet, a compression bandage, and low-suction pressure drainage at $200 \mathrm{~mm} \mathrm{Hg}$ for 24 hours. For 4 days after surgery, the analgesic regimen included acetaminophen $1 \mathrm{~g} 4$ times daily and sustained-release oral diclofenac $100 \mathrm{mg}$ daily for 4 days. Patients were provided a patient-controlled analgesia pump with intravenous morphine. Continuous femoral nerve block was performed. Patients were reviewed by Acute Pain Service staff beginning on postoperative day 0 . The complete blood count was checked on postoperative day 1 . For patients with a haemoglobin level $<8 \mathrm{~g} / \mathrm{dL}$, blood

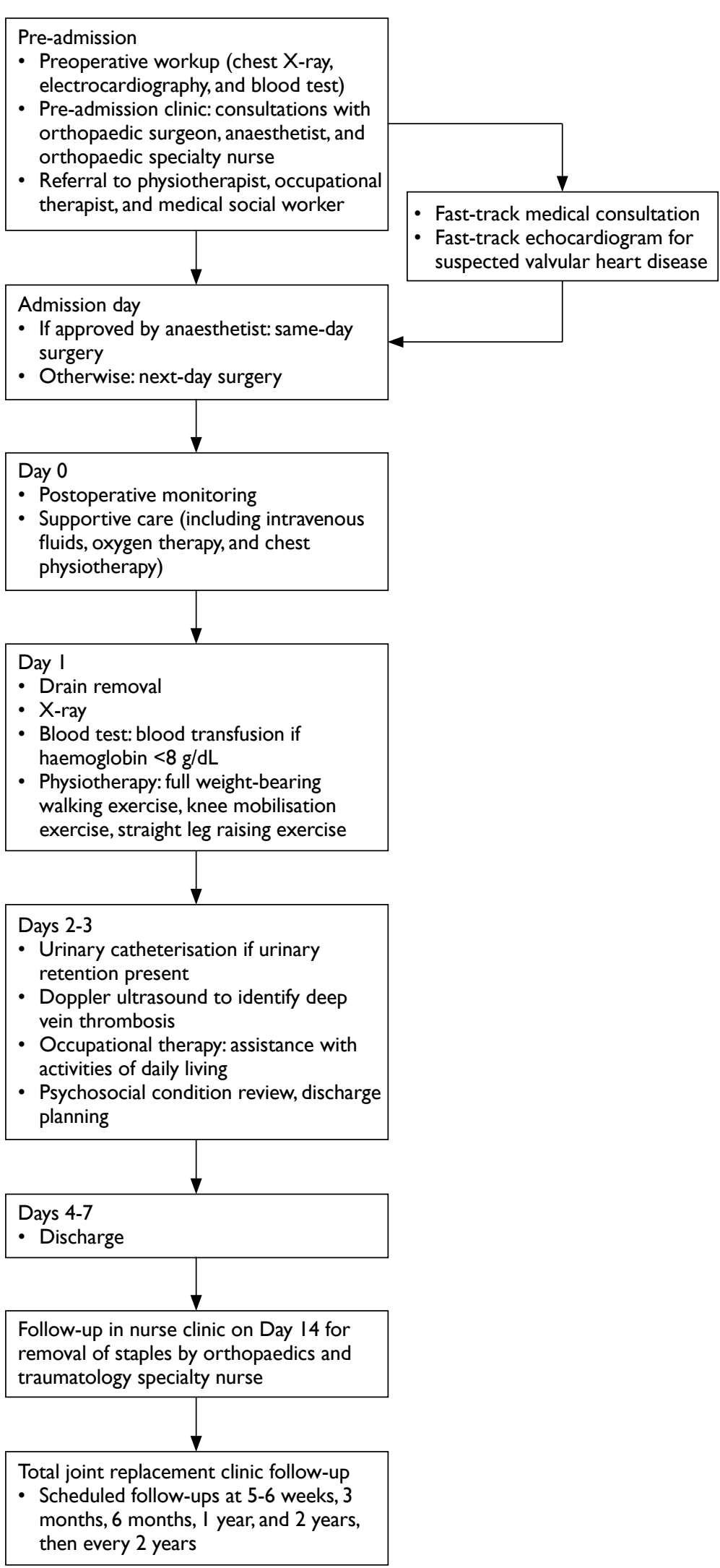

FIG I. Fast-track total knee arthroplasty pathway 


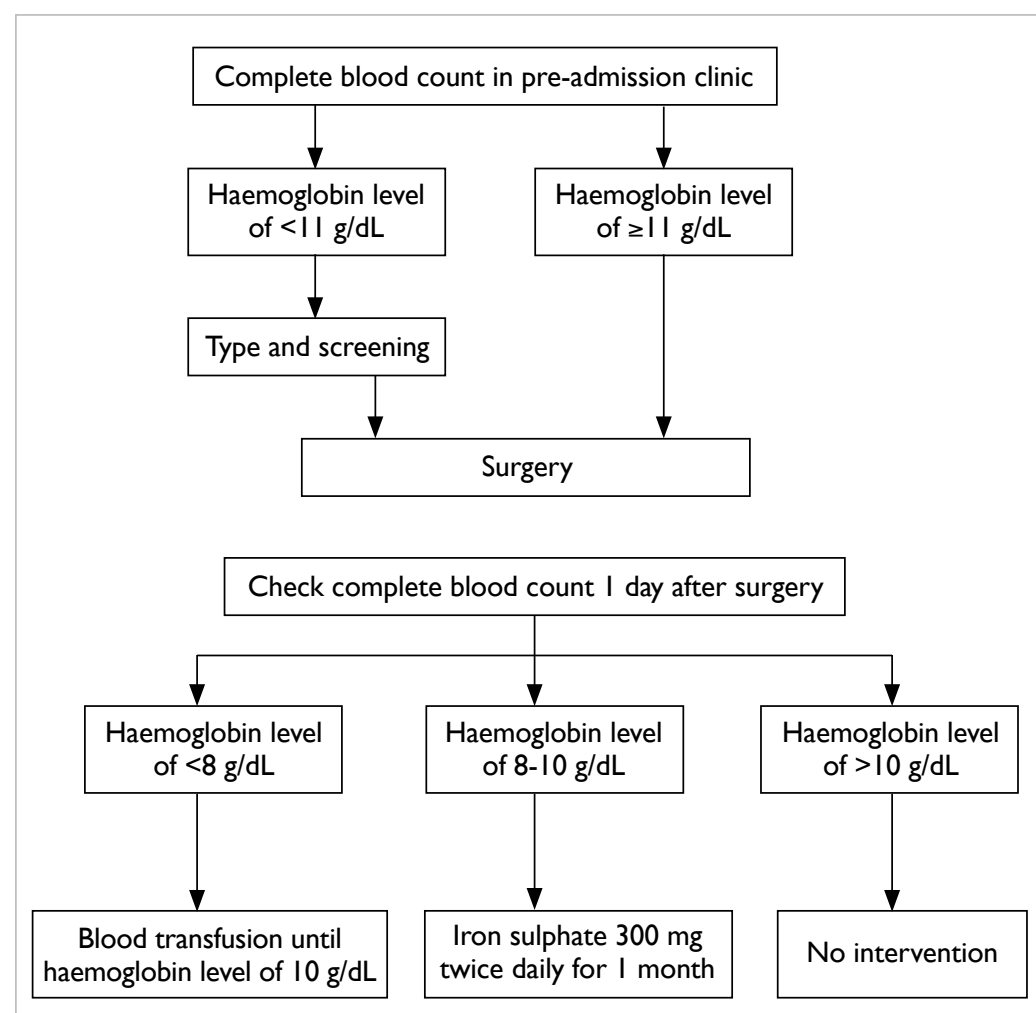

FIG 2. Protocol for perioperative blood transfusion in fast-track total knee arthroplasty 15 measurements. Univariate analysis was performed using the Chi squared test and unpaired $t$ tests, respectively, for categorical and numerical data with normal distributions. Comparisons of parameters with non-normal distributions were performed using the Mann-Whitney $U$ test. To adjust for the confounding effects of multiple variables (eg, type of anaesthesia, Charlson Comorbidity Index, and other baseline co-morbidities), multivariate analysis was performed with multiple logistic regressions. The high-risk group was defined as Charlson Comorbidity Index $>5$, on the basis of previous findings regarding the risk of complications in TKA. ${ }^{13}$ Data were reported as mean \pm standard deviation unless otherwise specified. Statistical significance was defined as $\mathrm{P}<0.05$. In addition, power analysis to determine the sample size was performed using G*Power (version 3.1.9.1), ${ }^{14}$ assuming that power $>0.80$ was indicative of an appropriate sample size.

\section{Results}

\section{Patient characteristics}

In total, 1788 patients underwent primary TKA during the study period (Table 1 ). Of these patients, $112(6.3 \%)$ were aged $>80$ years (octogenarian group; mean age, $82.7 \pm 1.6$ years; range, 81-89); 80 patients $(71.4 \%)$ in the octogenarian group were women. From the remaining patients, 108 sex- and BMI-matched patients aged $\leq 80$ years (mean age, $66.4 \pm 8.7$ years; range, 43-80) were selected at random and assigned to the control group (ie, nonoctogenarian group). The mean follow-up interval for all patients was 3.2 years (range, $2-5$ years).

The indication for TKA in most patients (218 patients, 99.1\%) was primary osteoarthritis of the knee joint. Two patients $(0.9 \%)$ had rheumatoid arthritis of the knee joint. There was no significant difference between groups in the number of patients with rheumatoid arthritis. The octogenarian group had a significantly higher Charlson Comorbidity Index $(4.9 \pm 1.0$ vs $2.5 \pm 1.2, \mathrm{P}<0.001)$, along with higher incidences of renal impairment and congestive heart failure.

More cases were performed with spinal anaesthesia in both groups. The ratio of general to spinal anaesthesia was lower in the octogenarian group (26:86 vs 40:68, $\mathrm{P}=0.025)$. Tourniquet time was significantly lower in the octogenarian group than in the non-octogenarian group $(89.20 \pm 17.25 \mathrm{mins}$ vs $99.62 \pm 23.94 \mathrm{~min}, \mathrm{P}=0.001)$. Preoperative and postoperative haemoglobin levels were both significantly lower in the octogenarian group. Both groups exhibited similar degrees of reduction in haemoglobin levels. More blood transfusions were recorded in the octogenarian group $(16.1 \%$ vs $0.9 \%$, $\mathrm{P}<0.001)$. 


\section{Length of stay and changes in functional outcomes}

The length of stay did not significantly differ between groups (octogenarian: $8.9 \pm 5.6 \mathrm{~d}$ vs nonoctogenarian: $7.5 \pm 2.6 \mathrm{~d}, \mathrm{P}=0.096)$ [Table 2]. Significantly more patients in the octogenarian group required a walking aid upon discharge (83.3\% vs $59.3 \%, \mathrm{P}<0.001)$. At 2 years after surgery, more patients in the octogenarian group continued to require a walking aid $(81.6 \%$ vs $35.8 \%, \mathrm{P}<0.001)$.

The octogenarian group exhibited a worse preoperative $\mathrm{ROM}\left(94 \pm 18^{\circ}\right.$ vs $\left.100 \pm 17^{\circ}, \mathrm{P}=0.009\right)$ and WOMAC score ( $48 \pm 20$ vs $53 \pm 17, \mathrm{P}=0.018$ ), but the improvements in these outcomes at 12 months after surgery were identical in both groups. In contrast, greater improvement in KSS was observed in the octogenarian group at 12 months after surgery (46 \pm 19 vs $39 \pm 16, \mathrm{P}=0.018)$, despite a similar preoperative score. Importantly, our sample size of 112 octogenarian patients and 108 non-octogenarian patients exhibited $83.7 \%$ power to detect a difference in the primary outcome of KSS improvement after surgery between groups when such a difference was present.

\section{Complications and mortality}

The incidence of major complications tended to be greater in the octogenarian group, although this difference was not statistically significant $(2.7 \%$ vs $0 \%, \mathrm{P}=0.087$ ) [Table 2]. The incidence of overall complications was significantly higher in the octogenarian group ( $15.2 \%$ vs $4.6 \%, \mathrm{P}=0.009)$. Despite the higher preoperative Charlson Comorbidity Index in the octogenarian group, there were no significant differences in the individual incidences of falls, urinary tract infection, proximal DVT, pulmonary embolism, confusion, and congestive heart failure, or in the rates of ICU admission and 1-year mortality. Notably, urinary catheterisation tended to occur more frequently in the octogenarian group, although this difference was not statistically significant $(11.6 \%$ vs $4.6 \%, \mathrm{P}=0.059$ ).

Logistic regression was performed to ascertain the effects of octogenarian status, Charlson Comorbidity Index $>5$, anaesthesia type, tourniquet time, preoperative ROM, and histories of multiple conditions (ie, renal impairment, congestive heart failure, major cerebrovascular accident, ischaemic heart disease, and peptic ulcer disease) on the likelihood that patients would experience complications after fast-track TKA. The model explained 26.7\% (Nagelkerke $R^{2}$ ) of the variance in complications and correctly classified $90.0 \%$ of patients. Patients with Charlson Comorbidity Index $>5$ were 5.69 -fold more likely to exhibit complications than were patients with a Charlson Comorbidity Index $\leq 5 \quad(\mathrm{P}=0.039 ;$ odds ratio $[\mathrm{OR}]=5.69$;
TABLE I. Demographic characteristics and surgical data of patients selected from I 788 patients who underwent total knee arthroplasty between $201 \mathrm{I}$ and $2015^{*}$

\begin{tabular}{|c|c|c|c|}
\hline & $\begin{array}{l}\text { Octogenarian } \\
\text { group }(n=112)\end{array}$ & $\begin{array}{l}\text { Non- } \\
\text { octogenarian } \\
\text { group }(n=108)\end{array}$ & P value \\
\hline Age, y & $82.7 \pm 1.6$ & $66.4 \pm 8.7$ & $<0.001^{\dagger}$ \\
\hline Age range, y & $81-89$ & $43-80$ & \\
\hline Male:female ratio & $32: 80$ & $30: 78$ & 0.896 \\
\hline Mean BMI, kg/m² & $27.0 \pm 4.2$ & $27.5 \pm 3.9$ & 0.410 \\
\hline Charlson Comorbidity Index & $4.9 \pm 1.0$ & $2.5 \pm 1.2$ & $<0.001^{\ddagger}$ \\
\hline \multicolumn{4}{|l|}{ Co-morbidity } \\
\hline Hypertension & $85(75.9 \%)$ & $73(67.6 \%)$ & 0.171 \\
\hline Diabetes mellitus & $27(24.1 \%)$ & $22(20.4 \%)$ & 0.505 \\
\hline Hyperlipidaemia & $34(30.4 \%)$ & $34(31.5 \%)$ & 0.857 \\
\hline Minor CVA & $9(8.0 \%)$ & $4(3.7 \%)$ & 0.173 \\
\hline Major CVA & 0 & $2(1.9 \%)$ & 0.240 \\
\hline Dementia & $1(0.9 \%)$ & 0 & 1.000 \\
\hline Renal impairment & $11(9.8 \%)$ & $2(1.9 \%)$ & $0.012^{\S}$ \\
\hline Benign prostatic hyperplasia & $13(11.6 \%)$ & $7(6.5 \%)$ & 0.186 \\
\hline COAD or asthma & $5(4.5 \%)$ & $2(1.9 \%)$ & 0.446 \\
\hline Obstructive sleep apnoea & $4(3.6 \%)$ & 0 & 0.122 \\
\hline Congestive heart failure & $10(8.9 \%)$ & $1(0.9 \%)$ & $0.006^{\prime \prime}$ \\
\hline Atrial fibrillation & $3(2.7 \%)$ & $1(0.9 \%)$ & 0.622 \\
\hline Ischaemic heart disease & $13(11.6 \%)$ & $6(5.6 \%)$ & 0.110 \\
\hline Peripheral vascular disease & $1(0.9 \%)$ & 0 & 1.000 \\
\hline Peptic ulcer & $14(12.5 \%)$ & $7(6.5 \%)$ & 0.129 \\
\hline Cancer in remission & $3(2.7 \%)$ & $2(1.9 \%)$ & 1.000 \\
\hline Cancer with active treatment & 0 & 0 & - \\
\hline Rheumatoid arthritis & $1(0.9 \%)$ & $1(0.9 \%)$ & 1.000 \\
\hline GA:SA ratio & $26: 86$ & $40: 68$ & $0.025^{\S}$ \\
\hline Tourniquet time, min & 89.20 & 99.62 & $0.001^{\dagger}$ \\
\hline $\mathrm{Hb}$ before surgery, g/dL & $12.4 \pm 1.5$ & $13.4 \pm 1.4$ & $<0.001^{\dagger}$ \\
\hline $\mathrm{Hb}$ after surgery, g/dL & $9.8 \pm 1.6$ & $10.7 \pm 1.4$ & $<0.001^{\dagger}$ \\
\hline $\mathrm{Hb}$ reduction, g/dL & $2.7 \pm 1.2$ & $2.8 \pm 1.1$ & 0.526 \\
\hline Type and screen & $15.2 \%$ & $4.6 \%$ & $0.009^{\prime \prime}$ \\
\hline Transfusion & $16.1 \%$ & $0.9 \%$ & $<0.001^{\|}$ \\
\hline
\end{tabular}

Abbreviations: $\mathrm{BMI}=$ body mass index; $\mathrm{COAD}=$ chronic obstructive airway disease; CVA = cerebrovascular accident; GA = general anaesthesia; $\mathrm{Hb}=$ haemoglobin level; SA = spinal anaesthesia

Data are shown as mean \pm standard deviation or No. (\%), unless otherwise specified Mann-Whitney $U$ test: $P<0.05$ was considered statistically significant for comparisons between two groups

Independent Student's $t$ test: $\mathrm{P}<0.0$ I was considered statistically significant for comparisons between two groups

Chi squared test: $\mathrm{P}<0.05$ was considered statistically significant for comparisons between two groups

Chi squared test: $\mathrm{P}<0.01$ was considered statistically significant for comparisons between two groups 
TABLE 2. Postoperative findings and follow-up assessments*

\begin{tabular}{|c|c|c|c|}
\hline & $\begin{array}{l}\text { Octogenarian } \\
\text { group }(n=112)\end{array}$ & $\begin{array}{c}\text { Non- } \\
\text { octogenarian } \\
\text { group }(n=108)\end{array}$ & $P$ value \\
\hline LOS, d & $8.9 \pm 5.6$ & $7.5 \pm 2.6$ & 0.096 \\
\hline \multicolumn{4}{|l|}{ Walking status } \\
\hline On discharge, aided & $83.3 \%$ & $59.3 \%$ & $<0.001^{\dagger}$ \\
\hline At 2 years after surgery, aided & $81.6 \%$ & $35.8 \%$ & $<0.001^{\dagger}$ \\
\hline \multicolumn{4}{|l|}{ ROM, degrees } \\
\hline Before surgery & $94 \pm 18^{\circ}$ & $100 \pm 17^{\circ}$ & $0.009^{\ddagger}$ \\
\hline 12 Months after surgery & $103 \pm 10^{\circ}$ & $106 \pm 13^{\circ}$ & $0.033^{\S}$ \\
\hline Improvement & $10 \pm 19^{\circ}$ & $6 \pm 15^{\circ}$ & 0.195 \\
\hline \multicolumn{4}{|l|}{ KSS } \\
\hline Before surgery & $48 \pm 16$ & $51 \pm 14$ & 0.130 \\
\hline 12 Months after surgery & $92 \pm 8$ & $91 \pm 10$ & 0.790 \\
\hline Improvement & $46 \pm 19$ & $39 \pm 16$ & $0.018^{\| \prime}$ \\
\hline \multicolumn{4}{|l|}{ WOMAC } \\
\hline Before surgery & $48 \pm 20$ & $53 \pm 17$ & $0.018^{\|}$ \\
\hline 12 Months after surgery & $78 \pm 14$ & $81 \pm 14$ & 0.133 \\
\hline Improvement & $29 \pm 22$ & $27 \pm 19$ & 0.486 \\
\hline Overall complications & $17(15.2 \%)$ & $5(4.6 \%)$ & $0.009^{\dagger}$ \\
\hline \multicolumn{4}{|l|}{ Major complications } \\
\hline Proximal DVT & $1(0.9 \%)$ & 0 & 1.000 \\
\hline Pulmonary embolism & $1(0.9 \%)$ & 0 & 1.000 \\
\hline Congestive heart failure & $1(0.9 \%)$ & 0 & 1.000 \\
\hline \multicolumn{4}{|l|}{ Minor complications } \\
\hline Fall & $2(1.8 \%)$ & 0 & 0.498 \\
\hline Urinary catheterisation & $13(11.6 \%)$ & $5(4.6 \%)$ & 0.059 \\
\hline Urinary tract infection & $1(0.9 \%)$ & 0 & 1.000 \\
\hline Confusion & $1(0.9 \%)$ & 0 & 1.000 \\
\hline ICU admission & $1(0.9 \%)$ & $1(0.9 \%)$ & 0.622 \\
\hline 1-Year mortality & $2(1.8 \%)$ & 0 & 0.498 \\
\hline
\end{tabular}

Abbreviations: DVT = proximal deep vein thrombosis; ICU = intensive care unit;

KSS = Knee Society Score; LOS = length of stay; ROM = range of motion;WOMAC =

Western Ontario and McMaster Universities Osteoarthritis Index

" Data are shown as mean \pm standard deviation or No. (\%), unless otherwise specified

Chi squared test: $\mathrm{P}<0.0$ I was considered statistically significant for comparisons between two groups

Independent Student's $t$ test: $P<0.01$ was considered statistically significant for comparisons between two groups

$\S$ Mann-Whitney $U$ test: $\mathrm{P}<0.0$ I was considered statistically significant for comparisons between two groups

"I Mann-Whitney $U$ test: $P<0.05$ was considered statistically significant for comparisons between two groups

\section{Discussion}

To our knowledge, this is the first study in Hong Kong concerning the clinical outcomes of fast-track TKA for patients aged $>80$ years. We found higher incidences of preoperative co-morbidities, anaemia, postoperative transfusion, and postoperative complications. Importantly, the improvements in functional outcomes at 1 year after fast-track TKA among octogenarians were comparable with or better than the improvements among younger patients. Our findings support the use of fasttrack TKA in this older group of patients; they indicate small and acceptable increases in the risks for such patients. This study focused on patient outcomes in our centre from 2011 to 2015. Because of protocol improvements in subsequent years, including opioid-sparing analgesia, local infiltrative anaesthesia, the use of oral tranexamic acid, and 1 year of physiotherapy, the length of stay and the patient outcomes are expected to improve.

\section{Haemoglobin reduction and blood product management}

In this study, the octogenarian group exhibited a lower preoperative haemoglobin level. The higher incidence of anaemia among octogenarians in the general population may be related to ageing, particularly because of reduced erythropoietin production, as well as anaemia secondary to iron, nutritional deficiency, or chronic disease. ${ }^{14}$ These factors presumably contributed to the significant increases in the rates of transfusion and 'type and screen' in the octogenarian group, despite a similar haemoglobin reduction and the use of $8 \mathrm{~g} / \mathrm{dL}$ as the transfusion threshold in both groups..$^{15}$ Because perioperative anaemia and allogenic blood transfusion have been associated with an increased risk of postoperative infection, longer hospital stay, and greater mortality, ${ }^{16}$ iron supplements and autologous transfusion were used to increase the preoperative haemoglobin level and reduce the postoperative transfusion rate. Our fast-track protocol-driven blood management with a single transfusion threshold provides a good balance between adequate treatment of perioperative anaemia and unnecessary blood transfusion. This balance was reflected by the absence of significant increases in periprosthetic infection and mortality in the octogenarian group.

\section{Length of stay}

95\% confidence interval $[\mathrm{CI}]=1.09-32.60)$. A history of major cerebrovascular accident $(\mathrm{P}=0.016 ; \mathrm{OR}=45.03$; 95\% $\mathrm{CI}=2.05-991.54)$ and a history of peptic ulcer disease $(\mathrm{P}=0.007 ; \quad \mathrm{OR}=5.51 ; 95 \% \quad \mathrm{CI}=1.58-19.17)$ were also significantly associated with an increased likelihood of exhibiting complications (Table 3).
The length of stay tended to be greater in the octogenarian group in the present study. Maiorano et $\mathrm{al}^{17}$ suggested that the mean length of stay was shorter in patients with a higher modified Barthel Index Score; better functional status may lead to more rapid rehabilitation progress. Furthermore, 
TABLE 3. Multivariate analysis of the risks of complications after fast-track total knee arthroplasty with octogenarian status after adjustment for confounding factors

\begin{tabular}{lccccc}
\hline Variables & $\begin{array}{c}\text { Regression } \\
\text { coefficient }\end{array}$ & SE & $\begin{array}{c}\text { Wald } \\
\text { statistic }\end{array}$ & P value* & OR (95\% Cl) \\
\hline Octogenarian status & 1.203 & 0.674 & 3.185 & 0.074 & $3.329(0.889-12.476)$ \\
\hline Charlson Comorbidity Index $>5$ & 1.785 & 0.867 & 4.244 & $0.039^{\dagger}$ & $5.692(1.091-32.598)$ \\
\hline Anaesthetic type (general anaesthesia) & 0.055 & 0.572 & 0.009 & 0.923 & $1.057(0.344-3.244)$ \\
\hline Tourniquet time & 0.013 & 0.014 & 0.851 & 0.356 & $1.013(0.986-1.040)$ \\
\hline Preoperative ROM & 0.002 & 0.014 & 0.029 & 0.865 & $1.002(0.975-1.031)$ \\
\hline Renal impairment & 0.211 & 1.022 & 0.043 & 0.836 & $1.235(0.167-9.148)$ \\
Congestive heart failure & -1.399 & 1.330 & 1.107 & 0.293 & $0.247(0.018-3.344)$ \\
\hline Major cerebrovascular disease & 3.807 & 1.577 & 5.825 & $0.016^{\dagger}$ & $45.034(2.045-991.535)$ \\
Ischaemic heart disease & -0.989 & 0.988 & 1.002 & 0.317 & $0.372(0.054-2.579)$ \\
\hline Peptic ulcer disease & 1.706 & 0.637 & 7.178 & $0.007^{\ddagger}$ & $5.505(1.581-19.171)$ \\
\hline
\end{tabular}

Abbreviations: $\mathrm{Cl}=$ confidence interval; $\mathrm{OR}=$ odds ratio; $\mathrm{ROM}=$ range of motion; $\mathrm{SE}=$ standard error

A binomial logistic was used to determine the $P$ value of this model

$P<0.05$ was considered statistically significant

$\mathrm{P}<0.0$ I was considered statistically significant

we observed a better preoperative WOMAC score in younger patients $(53 \pm 17$ vs $48 \pm 20, \mathrm{P}<0.05)$, consistent with the findings by Maiorano et al. ${ }^{17} \mathrm{~A}$ phenomenon unique to Hong Kong is the confined living area in most homes, which may increase the difficulty in using a walking aid at home. In the present study, $83.3 \%$ of patients in the octogenarian group required a walking aid upon discharge. Additionally, temporary residential service was required more frequently upon discharge; this arrangement might have contributed to the increased length of stay. However, prior anticipation of discharge difficulty and the multidisciplinary approach in our fasttrack TKA protocol helped to limit the length of stay, leading to an increase of only 1.4 days in the octogenarian group.

\section{Functional outcomes}

In this study, both groups had comparable outcomes at 12 months after surgery in terms of improvements in ROM and WOMAC score. The octogenarian group exhibited greater improvement in KSS at 12 months after surgery. These results support the use of TKA among octogenarian patients. Good functional outcomes without pain are important for ensuring that patients maintain independence in the activities of daily living. Such independence relieves the caretaker burden and helps patients return to the community.

\section{Mortality and complication rate}

In this analysis of fast-track TKA, there was no significant difference between octogenarian and non-octogenarian groups in terms of the 1-year mortality rate, although the octogenarian group had a higher Charlson Comorbidity Index. Notably, the all-cause mortality rate within 1 year after surgery was $1.79 \%$ in the octogenarian group; this was lower than the annual all-cause mortality rates for the Hong Kong general population in 2013 among men and women aged 80 to 84 years $\left(6.1 \%\right.$ and $3.7 \%{ }^{18}$, respectively). In the fast-track protocol, all patients were assessed by an anaesthetist, an orthopaedic surgeon, and an orthopaedic nurse. Patients with suboptimally controlled medical condition were rapidly referred for out-patient treatment by the appropriate department. For instance, fast-track echocardiography was arranged for patients with suspected valvular problems; fast-track management of poorly controlled hypertension was performed by family medicine specialists.

Significantly more patients in the octogenarian group developed complications. Most patients exhibited minor complication. There were no significant differences between groups in terms of major complications (eg, proximal DVT, pulmonary embolism, and congestive heart failure). Furthermore, postoperative confusion was rare, in contrast to the incidence rate of $6.7 \%$ reported by Kuo et al. ${ }^{19}$ Postoperative maintenance of good pain control and normal cognitive status is crucial for rehabilitation and ensuring safety. ${ }^{20} \mathrm{~A}$ multimodel analgesic regimen in the 4 days after surgery was implemented in our centre to achieve the greatest degree of analgesia with the fewest side-effects. Each patient was provided patient-controlled analgesia comprising morphine infusion, paracetamol, and nonsteroidal anti-inflammatory drugs.

After adjustment for confounding factors, 
patients with Charlson Comorbidity Index $>5$ were 5.69-fold more likely to develop postoperative complications. The Charlson Comorbidity Index has been widely used in large studies to predict functional outcome, implant survival, mortality, and length of stay after TKA..$^{21,22}$ Our results were consistent with the findings by Marya et $\mathrm{al}^{13}$ that a Charlson Comorbidity Index of $>5$ was associated with major complications after bilateral simultaneous TKA. In addition, we found that patients with past histories of major cerebrovascular accident and peptic ulcer disease were more likely to have postoperative complications. Previous stroke has been identified as a predictive factor for perioperative acute ischaemic stroke after TKA, ${ }^{23}$ whereas peptic ulcer disease has been associated with periprosthetic fracture after primary TKA. ${ }^{24}$ After adjustment for confounding factors, we found that octogenarian status alone was not associated with significantly greater risk of complications after fast-track TKA. Surgeons should consider patient risk during TKA on the basis of their individual co-morbidities, rather than age alone.

\section{Urinary catheterisation}

There was a considerably higher incidence of urinary catheterisation in the octogenarian group. Lingaraj et $\mathrm{al}^{25}$ suggested that $8 \%$ of all patients undergoing TKA develop urinary retention. There is a need to identify postoperative urinary retention to reduce the risks of periprosthetic joint infection and renal impairment. As part of our fast-track protocol, a protocol-driven management approach was used for each patient with acute urine retention. Bladder scans were performed by nurses to assess postmicturition volume; catheterisation was performed in patients with any bladder distention. Stimulant laxative treatment was used to avoid constipation and alleviate urine retention.

\section{Limitations}

There were some limitations in this study. First, the study was small and the follow-up period was short (mean, 3.2 years). However, there are generally few patients aged $>80$ years who have sufficient life expectancy for a longer period of follow-up. Second, this was a retrospective cohort study, with the inherent limitations of the retrospective design. Although a prospective randomised controlled trial is preferable, ethical considerations prohibit the allocation of octogenarian patients to a non-fasttrack protocol because of their higher operative risks. Third, although coronal plane deformity and the degree of soft tissue balance may contribute to differences in knee functional performance, these parameters were not measured. Future studies should include such assessments to more fully characterise the factors that influence TKA outcomes.

\section{Conclusion}

Compared with non-octogenarians, octogenarians had greater improvement in KSS at 1 year after fast-track TKA, despite similar preoperative KSS. Octogenarians had a higher incidence of complications after TKA. After adjustment for confounding factors, we found that Charlson Comorbidity Index $>5$, history of major cerebrovascular accident, and history of peptic ulcer disease were predictive of complications after fasttrack TKA; importantly, octogenarian status was not predictive of complications. There were no significant differences in the length of hospitalisation, incidence of major complications, rate of ICU admission, or the 1-year mortality rate between the octogenarian and non-octogenarian groups. Thus, age alone should not be a contra-indication to TKA.

\section{Author contributions}

Concept or design: All authors.

Acquisition of data: CH Lee, EWY Chang.

Analysis or interpretation of data: $\mathrm{CH}$ Lee, EWY Chang.

Drafting of the manuscript: TP Leung, $\mathrm{CH}$ Lee, QJ Lee.

Critical revision of the manuscript for important intellectual content: All authors.

All authors had full access to the data, contributed to the study, approved the final version for publication, and take responsibility for its accuracy and integrity.

\section{Conflicts of interest}

All authors have disclosed no conflicts of interest.

\section{Funding/support}

This research received no specific grant from any funding agency in the public, commercial, or not-for-profit sectors.

\section{Ethics approval}

This study was approved by the Kowloon West Cluster Research Ethics Committee (Ref: KW/EX-20-068(147-03)).

\section{References}

1. Census and Statistics Department, Hong Kong SAR Government. Hong Kong Population Projection 20122041. Available from: https://www.censtatd.gov.hk/ media_workers_corner/pc_rm/hong_kong_population_ projections_2012_2041/index.jsp. Accessed 31 Jul 2012.

2. Yuen W. Osteoarthritis of knees: the disease burden in Hong Kong and means to alleviate it. Hong Kong Med J 2014;20:5-6.

3. Kreder HJ, Berry GK, Mcmurtry IA, Halman SI. Arthroplasty in the octogenarian: quantifying the risks. J Arthroplasty 2005;20:289-93.

4. Jämsen E, Puolakka T, Eskelinen A, et al. Predictors of mortality following primary hip and knee replacement in the aged. A single-center analysis of 1998 primary hip and knee replacements for primary osteoarthritis. Acta Orthop 2012;84:44-53.

5. Scott JE, Mathias JL, Kneebone AC. Postoperative cognitive 
dysfunction after total joint arthroplasty in the elderly: a meta-analysis. J Arthroplasty 2014;29:261-7.e1.

6. Mnatzaganian G, Ryan P, Norman PE, Davidson DC, Hiller JE. Total joint replacement in men: old age, obesity and in-hospital complications. ANZ J Surg 2012;83:37681.

7. Klasan A, Putnis SE, Yeo WW, Fritsch BA, Coolican MR, Parker DA. Advanced age is not a barrier to total knee arthroplasty: a detailed analysis of outcomes and complications in an elderly cohort compared with average age total knee arthroplasty patients. J Arthroplasty 2019;34:1938-45.

8. Petruccelli D, Rahman WA, de Beer J, Winemaker M. Clinical outcomes of primary total joint arthroplasty among nonagenarian patients. J Arthroplasty 2012;27:1599-603.

9. Shah AK, Celestin J, Parks ML, Levy RN. Long-term results of total joint arthroplasty in elderly patients who are frail. Clin Orthop Relat Res 2004;425:106-9.

10. Hernández-Vaquero D, Fernández-Carreira JM, PérezHernández D, Fernández-Lombardía J, García-Sandoval MA. Total knee arthroplasty in the elderly. Is there an age limit? J Arthroplasty 2006;21:358-61.

11. Joshi AB, Markovic L, Gill G. Knee arthroplasty in octogenarians: results at 10 years. J Arthroplasty 2003;18:295-8

12. Berend ME, Thong AE, Faris GW, Newbern G, Pierson JL, Ritter MA. Total joint arthroplasty in the extremely elderly: hip and knee arthroplasty after entering the 89th year of life. J Arthroplasty 2003;18:817-21.

13. Marya SK, Amit P, Singh C. Impact of Charlson indices and comorbid conditions on complication risk in bilateral simultaneous total knee arthroplasty. Knee 2016;23:955-9.

14. Partridge J, Harari D, Gossage J, Dhesi J. Anaemia in the older surgical patient: a review of prevalence, causes, implications and management. J R Soc Med 2013;106:26977.

15. Lee QJ, Mak WP, Yeung ST, Wong YC, Wai YL. Blood management protocol for total knee arthroplasty to reduce blood wastage and unnecessary transfusion. J Orthop Surg (Hong Kong) 2015;23:66-70.

16. Spahn DR. Anemia and patient blood management in hip and knee surgery: a systematic review of the literature. Anesthesiology 2010;113:482-95.

17. Maiorano E, Bodini BD, Cavaiani F, Pelosi C, Sansone V. Length of stay and short-term functional outcomes after total knee arthroplasty: can we predict them? Knee 2017;24:116-20.

18. Census and Statistics Department, Hong Kong SAR Government. Hong Kong Monthly Digest of Statistics November 2014. The mortality trend in Hong Kong, 1981 to 2013. Available from: https://www.statistics.gov.hk/pub/ B71411FB2014XXXXB0100.pdf. Accessed 17 Nov 2014.

19. Kuo FC, Hsu CH, Chen WS, Wang JW. Total knee arthroplasty in carefully selected patients aged 80 years or older. J Orthop Surg Res 2014;9:61.

20. Lynch EP, Lazor MA, Gellis JE, Orav J, Goldman L, Marcantonio ER. The impact of postoperative pain on the development of postoperative delirium. Anesth Analg 1998;86:781-5

21. Kreder HJ, Grosso P, Williams JI, et al. Provider volume and other predictors of outcome after total knee arthroplasty: a population study in Ontario. Can J Surg 2003;46:15-22.

22. Bjorgul K, Novicoff WM, Saleh KJ. Evaluating comorbidities in total hip and knee arthroplasty: available instruments. J Orthop Traumatol 2010;11:203-9.

23. Menendez ME, Greber EM, Schumacher CS, Lowry Barnes C. Predictors of acute ischemic stroke after total knee arthroplasty. J Surg Orthop Adv 2017;26:148-53.

24. Singh JA, Lewallen DG. Association of peptic ulcer disease and pulmonary disease with risk of periprosthetic fracture after primary total knee arthroplasty. Arthritis Care Res (Hoboken) 2011;63:1471-6.

25. Lingaraj K, Ruben M, Chan YH, Das SD. Identification of risk factors for urinary retention following total knee arthroplasty: a Singapore hospital experience. Singapore Med J 2007;48:213-6. 